\title{
Analysis, Simulation and Control of a New Measles Epidemic Model
}

\author{
Paolo Di Giamberardino and Daniela Iacoviello \\ Dept. Computer, Control and Management Engineering Antonio Ruberti, Sapienza University of Rome, \\ via Ariosto 25, 00185 Rome, Italy
}

Keywords: Epidemic Modeling, System Analysis, Optimal Control.

Abstract: In this paper the problem of modeling and controlling the measles epidemic spread is faced. A new model is proposed and analysed; besides the categories usually considered in measles modeling, the susceptible, the exposed, the infected, the removed and, less frequently, the quarantine individuals, two new categories are herein introduced: the immunosuppressed subjects, that can not be vaccinated, and the patients with an additional complication, not risky by itself but dangerous if caught togeter with the measles. These two novelties are taken into account in designing and scheduling suitably control actions such as vaccination, whenever possible, prevention, quarantine and treatment, when limited resources are available. An analysis of the model is developed and the optimal control strategies are compared with other not optimized actions. By using the Pontryagin principle, it is shown the prevailing role of the vaccination in guaranteeing the protection to immunosuppressed individuals, as well as the importance of a prompt response of the society when an epidemic spread occurs, such as the quarantine intervention.

\section{INTRODUCTION}

Despite a vaccination of the measles exists since 1963, it is still an important cause of death, especially among young children. The measles is caused by a virus that infects the respiratory tract and then spreads all over the body; it is highly contagious: it is transmitted by infected patients through droplets from nose, mouth and throat. After about 10 days from the infection the main symptoms are high fever, bloodshot eyes and tiny white spots inside the mouth; then, a rash spread all over the body, starting from the face. Generally, for good healthy people, the measles does not represents a serious illness; it becomes risky if the infected subjects is a poorly nourished children (age less than 5 years old) with insufficient vitamin A or subjects with the immune system weakened by chemotherapy, or HIV/AIDS or other diseases. In these cases complications may lead to death; the most commons are: diarrhoea (about $70 \%$ of the hospitalized persons with measles in the US), pneumonia (more than 9\% among children with less than 5 years old) and other respiratory infections (more than $50 \%$ among hospitalized children with measles), encephalitis (mainly occurring in patients with HIV infection or leukaemia, ocular complications (R.T.Perry and N.A.Halset, 2004).
Mathematical modeling of epidemic diseases allows to describe and analyse epidemic diseases, (Nowzari et al., 2016), (Nowak and May, 2000), (Pinto and Rocha, 2012), (Naresh et al., 2009), (Di Giamberardino et al., 2018),(Kuniya and Nakata, 2012), (TW et al., 2003). The availability of suitable model allows to determine the most effective control strategy and resources allocation, (Di Giamberardino and Iacoviello, 2018a), (X.Yan and Y.Zou, 2008), (Di Giamberardino and Iacoviello, 2017), (Ledzewicz and Schattler, 2011), (Iacoviello and Stasio, 2013). The most common models for the measles uses the SEIR representation, where $S$ stands for the Susceptible individuals, that are the healthy people; $E$ represents the compartment of the Exposed, that are the subjects infected but not yet infectious; $I$ is the compartment of infected and infectious patients; $R$ contains the subjects Recovered, including the individuals that have got the immunity by vaccination or having got the measles previously, (A.A.Momoh et al., 2013), (L.Pang et al., 2015), (O.O.Onyejekwe and E.Z.Kebede, 2015). Sometimes also the class of isolated subjects is introduced, (S.O.Adewale et al., 2016), that is the class $Q$ of subjects induced to isolation to avoid new infections; in this framework it is shown the existence of a unique endemic equilibrium point as well as the most efficiency of the vaccina- 
tion control strategy with respect to the treatment and the campaign aiming at educating the society to manage the measles infection. Another interesting improvement of the classical SEIR model is proposed in (S.Verguet et al., 2015) where the Exposed individuals are not considered, stressing the subjects vaccinated susceptible, vaccinated infected and vaccinated recovered, taking into account the risks of measles infection also for vaccinated subjects. An interesting study that takes into account the life histories of interacting individuals is proposed in (E.A.J.Fischer et al., 2008), where a general approach that could be applied also to the measles disease is discussed; it is based on microsimulation models, considering birth, death and general demographic events. The possibility of taking into account the complications is considered in (Di Giamberardino and Iacoviello, 2018b), where a general infectious disease (not necessarily the measles) is considered, and suitable control strategies for an efficient resources allocation are proposed. In this paper, it is stressed that some subjects in the population could not be vaccinated, for example the immunosuppressed people; moreover it is considered that the patients infected by the measles are particularly exposed to complications, dangerous for weak people and/or young children. The model introduced takes into account the different peculiarities in a susceptible population: two categories of susceptible subjects are introduced, distinguishing among healthy individuals that can or cannot be vaccinated. Then, it is included the classical categories of $E x$ posed; as in (S.O.Adewale et al., 2016), it is considered the category of isolated subjects $Q$. The category of Infected patients is split in order to consider the subjects infected by the infectious disease and those that have caught also the complication. Finally there is the class of Removed people. The possible control strategies introduced to face in particular the presence of subjects that can not be vaccinated as well as the possibility of complications include the following actions:

- prevention actions, such as vaccination (when possible) and informative campaign;

- treatment control to those patients that have got the infection;

- treatment control to those subjects that have got also the second not infectious disease.

The paper is organized as follows; Section 2 is divided into three subsections: in the first two, the considered model is deeply described and analysed discussing the existence of the equilibrium points. In the third Subsection optimal control strategies are introduced. In Section 3 numerical results and discussion are pro- posed; conclusions and future developments are summarized in Section 4.

\section{MATERIALS AND METHODS}

In this Section a new model of the measles spread is described, Subsection 2.1, and analysed, Subsection 2.2. In Subsection 2.3 the optimal control strategy is proposed.

\subsection{The Mathematical Model}

The mathematical model here analysed takes into account the most important issues of the measles:

- the possibility that some subjects in the population could not be vaccinated and therefore it is advisable to get the herd immunity;

- the risky condition of having also a complication along with the infectious disease.

To this aim seven classes of people are introduced: the Susceptible subjects $S 1$, healthy people not infected; the Susceptible subjects $S_{2}$, healthy people not infected that cannot be vaccinated; the Exposed individuals $E$, infected people, not yet infectious; the Quarantine patients $Q$, patients that from the Exposed class are isolated; the Infected patients $I_{1}$, that may transmit the virus; the Infected patients $I_{2}$, that may transmit the virus and with also the non infectious complication; the Recovered people $R$, that have been immunized by vaccine or have been recovered from the $I_{1}$ or the $I_{2}$ stage. Note that the $Q$ patients get the measles, they become infected and infectious, but they cannot infect any other susceptible subject

In Fig. 1 it is shown the block diagram of the proposed model. The control actions introduced include

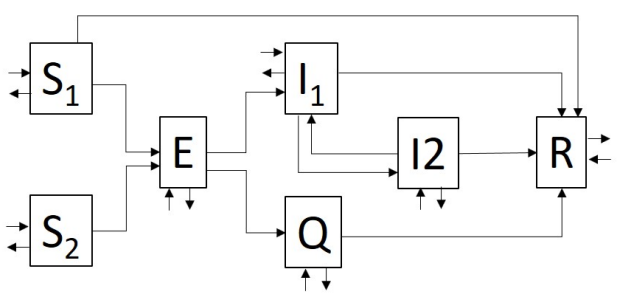

Figure 1: Block diagram of the proposed model.

preventions and treatments; among the former the predominant one is the vaccination that could be applied only on the $S_{1}$ individuals. The treatments include both the actions to face the infectious disease and the complication; also the patients in the quarantine compartment could be considered the effect of a treatment, meaning that they could not infect any 
susceptible individual. Therefore the proposed model could be described by the following differential equations:

$$
\begin{aligned}
\dot{S}_{1}= & b_{1}-d_{1} S_{1}-\beta_{11} S_{1} I_{1}-\beta_{12} S_{1} I_{2} \\
& +\delta_{2} u_{p 1} S_{1}-\delta_{1} u_{V} S_{1} \\
\dot{S}_{2}= & b_{2}-d_{2} S_{2}-\beta_{21} S_{2} I_{1}-\beta_{22} S_{2} I_{2} \\
& +\delta_{3} u_{p 2} S_{2} \\
\dot{E}= & b_{3}-d_{3} E+\beta_{11} S_{1} I_{1}+\beta_{12} S_{1} I_{2} \\
& +\beta_{21} S_{2} I_{1}+\beta_{22} S_{2} I_{2}-\delta_{4} E \\
\dot{Q}= & -d_{4} Q-\gamma_{3} Q+\delta_{4} u_{T 1} E-\delta_{5} u_{T 2} Q \\
\dot{I}_{1}= & b_{4}-d_{5} I_{1}+\delta_{4}\left(1-u_{T 1}\right) E-\gamma_{1} I_{1}-\sigma_{1} I_{1} \\
& +\sigma_{2} I_{2}-\delta_{6} u_{T 3} I_{1} \\
\dot{I}_{2}= & b_{5}-d_{6} I_{2}-\gamma_{2} I_{2}+\sigma_{1} I_{1}-\sigma_{2} I_{2} \\
& -\delta_{7} u_{T 4} I_{2} \\
\dot{R}= & b_{6}-d_{7} R+\gamma_{1} I_{1}+\gamma_{2} I_{2}+\gamma_{3} Q+\delta_{1} u_{V} S_{1} \\
& +\delta_{5} u_{T 2} Q+\delta_{6} u_{T 3} I_{1}+\delta_{7} u_{T 4} I_{2}
\end{aligned}
$$

where, using for notation' reasons the subscripts $1, \ldots, 6$ referring to quantities in the $S_{1}, S_{2}, E, I_{1}, I_{2}$ and $R$ respectively, $b_{i}, i=1, \ldots, 6$ is the rate of new people entering the compartments; $d_{j}, j=1, \ldots, 7$ is the removed rate from every compartments $S_{1}, S_{2}, E$, $Q, I_{1}, I_{2}$ and $R$ respectively, including also deaths; $\beta_{i j}$, $i=1,2 j=1,2$ is the contact rate between the subjects in the compartment $S_{i}$ and the ones in $I_{j} ; \sigma_{i}, i=1,2$ is the infection rate with respect to the complication disease involving the subjects in the $I_{1}$ and the $I_{2}$ compartments; $\gamma_{i}, i=1,2$ is the recovery rate from the $I_{1}$ and the $I_{2}$ compartment respectively; $\delta_{i}, i=1,2, . ., 7$ is related with the control success for the actions $u_{V}$, $u_{P i}, i=1,2$ and $u_{T j}, j=1, . ., 4$, respectively. Let us define the state vector:

$$
X=\left(\begin{array}{lllllll}
S 1 & S_{2} & E & Q & I_{1} & I_{2} & R
\end{array}\right)^{T}
$$

and the control vector:

$$
U=\left(\begin{array}{lllllll}
u_{V} & u_{P 1} & u_{P 2} & u_{T 1} & u_{T 2} & u_{T 3} & u_{T 4}
\end{array}\right)^{T}
$$

We introduce the vector $F$

$$
F=\left(\begin{array}{lllllll}
f_{1} & f_{2} & f_{3} & f_{4} & f_{5} & f_{6} & f_{7}
\end{array}\right)^{T}
$$

where the $f_{i}$ are the r.h.s. functions of equations (1)(7).

The proposed complete model with complications may be written in a compact form as follows:

$$
\dot{X}=F(X, \quad U, \quad t)
$$

starting from the initial conditions:

$$
X(0)=X_{0}
$$

The control actions are assumed bounded:

$$
0 \leq U_{i}(t) \leq U_{i}^{M}
$$

being $U_{i}$ the i- th component of the control $U$ given by (9) and $U_{i}^{M}$ its corresponding possible maximum value. In particular, note that for the $u_{T 1}$ control the maximum value allowed could be no more than 1 .

A subject in the $S_{1}$ compartment could be healthy all life long, or, if vaccinated, he could reach the recovered class $R$ or he could become exposed and therefore get the infectious disease and, eventually, the complication. For a subject in the $S_{2}$ class the best condition is to remain in that class, since the only other possibility is to get the infection and maybe also the risky complication. For the patients infected, but not yet infectious, two possibilities are allowed: to go in the quarantine compartment $Q$, thus avoiding to infect other $S_{1}$ and $S_{2}$ subjects, or to proceed in the $I_{1}$ and, maybe, in the $I_{2}$ classes. Note that the patients in the $Q$ class could get also the complication; they differ from the $I_{1}$ and $I_{2}$ patients since they can not infect any other susceptible individuals.

These considerations suggest the importance of a suitable control strategy and in particular to reach the herd immunity; it is important not only for the immunosuppressed subject, already weakened by other therapies, but for all the population since the complications in conjunction with the infectious disease could be fatal, especially for very young children and for old people. The analysis of the model with its equilibrium points is proposed in the next Subsection 2.2 .

\subsection{The Model Analysis}

In this Subsection the model (11) is analysed; the determination of the equilibrium points is not an easy task, due to the high number of parameters of the model. Nevertheless, some general considerations may be proposed and verified in the numerical results section.

To determine the equilibrium points in the model (11) the control inputs $U_{i}$ are assumed null, as well as the entries $b_{j}, j=3, \ldots, 6$. Therefore, the equation:

$$
F(X)=0
$$

must be solved; as obvious, it is always $Q^{e}=0$. As it can be easily verified, the point:

$$
P_{e 1}=\left(\begin{array}{lllllll}
b_{1} / d_{1} & b_{2} / d_{2} & 0 & 0 & 0 & 0 & 0
\end{array}\right)^{T}
$$

is always an equilibrium one.

To check the possible existence of other equilibrium points, the equation (6) is considered by imposing its equality to zero; it can be deduced that the numbers of infected subjects $I_{1}$ and $I_{2}$ patients in the equilibrium point (if it exists) are proportional:

$$
I_{1}^{e}=m_{1} I_{2}^{e}
$$


having defined

$$
m_{1}=\frac{\left(\sigma_{2}+\gamma_{2}+d_{6}\right)}{\sigma_{1}}>0
$$

From equation (7) it is deduced:

$$
R^{e}=m_{2} I_{2}^{e}
$$

with

$$
m_{2}=\frac{m_{1} \gamma_{1}+\gamma_{2}}{d_{7}}>0
$$

Analogously, equation (5) allows to write

$$
E^{e}=m_{3} I_{2}^{e}
$$

with

$$
m_{3}=\frac{\left(d_{5}+\gamma_{1}+\sigma_{1}\right) m_{1}-\sigma_{2}}{\delta_{4}}>0
$$

It is important to stress that from equation (1) it can be deduced that if an acceptable value for the equilibrium $I_{2}^{e}$ exists, then also $S_{1}^{e}$ is defined; in fact

$$
S_{1}^{e}=\frac{b_{1}}{\left[d_{1}+\left(\beta_{11} m_{1}+\beta_{12}\right) I_{2}^{e}\right]}
$$

Analogously, also $S_{2}^{e}$ is well defined once $I_{2}^{e}$ is, as it is deduced from (2)

$$
S_{2}^{e}=\frac{b_{2}}{\left[d_{2}+\left(\beta_{21} m_{1}+\beta_{22}\right) I_{2}^{e}\right]}
$$

Note that if $I_{2}^{e}$ is equal to zero the equilibrium point $P_{e 1}$ is determined. By summing (1), (2), (3) and substituting the expression (22) and (23), the following equation is determined:

$$
\begin{array}{r}
b_{1}+b_{2}-\frac{b_{1} d_{1}}{d_{1}+\left(\beta_{11}+m_{1}+\beta_{12}\right) I_{2}^{e}} \\
-\frac{b_{2} d_{2}}{d_{2}+\left(\beta_{21}+m_{1}+\beta_{22}\right) I_{2}^{e}} \\
=\frac{\left(\left(d_{5}+\gamma_{1}+\sigma_{1}\right) m_{1}-\sigma_{2}\right) d_{3} I_{2}^{e}}{\delta_{4}}
\end{array}
$$

By rearranging (24), if $I_{2}^{e} \neq 0$, a second order equation is obtained:

$$
A I_{2}^{e 2}+B I_{2}^{e}+C=0
$$

where

$$
\begin{aligned}
A= & d_{3}\left(\beta_{11} m_{1}+\beta_{12}\right)\left(\beta_{21} m_{1}+\beta_{22}\right)\left[\left(d_{5}+\gamma_{1}\right.\right. \\
& \left.\left.+\sigma_{1}\right) m_{1}-\sigma_{2}\right] \\
B= & {\left[\left(d_{5}+\gamma_{1}+\sigma_{1}\right) m_{1}-\sigma_{2}\right]\left[d _ { 1 } d _ { 3 } \left(\beta_{21} m_{1}\right.\right.} \\
& \left.\left.+\beta_{22}\right)+d_{2} d_{3}\left(\beta_{11} m_{1}+\beta_{12}\right)\right] \\
& -\left(\beta_{11} m_{1}+\beta_{12}\right)\left(\beta_{21} m_{1}+\beta_{22}\right)\left(b_{1}+b_{2}\right) \delta_{4}
\end{aligned}
$$

$$
\begin{aligned}
& C=d_{1} d_{2} d_{3}\left[\left(d_{5}+\gamma_{1}+\sigma_{1}\right) m_{1}-\sigma_{2}\right] \\
& -b_{1} d_{2} \delta_{4}\left(\beta_{11} m_{1}+\beta_{12}\right)-b_{2} d_{1} \delta_{4}\left(\beta_{21} m_{1}+\beta_{22}\right)
\end{aligned}
$$

The coefficient $A$ is always positive; to establish the sign of the equation (25) it necessary to check if both $B$ and $C$ can be positive (thus no real root is possible), or if there is one change of sign in the coefficients $A$, $B$ and $C$ (thus one positive root exists), or if there are two changes of sign (thus two positive roots exist). The latter case corresponds to the condition: $B<0$ and $C>0$, being $A$ always positive. In that case, from the definitions of (27) and (28) one has:

$$
\begin{array}{r}
\left(\beta_{11} m_{1}+\beta_{12}\right)\left(\beta_{21} m_{1}+\beta_{22}\right)\left(b_{1}+b_{2}\right) \delta_{4} \\
>\left[d_{1} d_{3}\left(\beta_{21} m_{1}+\beta_{22}\right)+d_{2} d_{3}\left(\beta_{11} m_{1}+\beta_{12}\right)\right] \\
{\left[\left(d_{1} d_{3}\right)^{-1} b_{1}\left(\beta_{11} m_{1}+\beta_{12}\right)+\right.} \\
\left.d_{2} d_{3}\right)^{-1} b_{2}\left(\beta_{21} m_{1}+\beta_{22}\right)
\end{array}
$$

which is absurd, resulting

$$
0>b_{1} d_{2} \frac{\left(\beta_{11} m_{1}+\beta_{12}\right)^{2}}{d_{1}}+b_{2} d_{1} \frac{\left(\beta_{21} m_{1}+\beta_{22}\right)}{d_{2}}
$$

Therefore, it can be concluded that it is not possible to have two solutions from equation (25).

The condition for which no root of (25) exists is verified once $B>0$ and $C>0$. From the definitions (27) and (28) it can be deduced that this occurs when:

$$
\left[\left(d_{5}+\gamma_{1}+\sigma_{1}\right) m_{1}-\sigma_{2}\right]>\max \left[B_{1}, C_{1}\right]
$$

where

$B_{1}=\frac{\left(\beta_{11} m_{1}+\beta_{12}\right)\left(\beta_{21} m_{1}+\beta_{22}\right)\left(b_{1}+b_{2}\right) \delta_{4}}{\left[d_{1} d_{3}\left(\beta_{21} m_{1}+\beta_{22}\right)+d_{2} d_{3}\left(\beta_{11} m_{1}+\beta_{12}\right)\right]}$

and

$C_{1}=\frac{b_{1} d_{2} \delta_{4}\left(\beta_{11} m_{1}+\beta_{12}\right)+b_{2} d_{1} \delta_{4}\left(\beta_{21} m_{1}+\beta_{22}\right)}{d_{1} d_{2} d_{3}}$

In the two situations discussed up to now no equilibrium point is determined from equation $(25)$ and the unique equilibrium point is $P_{e 1}$. Now we deduce the conditions under which one real positive solution exists of equation (25); it occurs when $B>0$ and $C<0$ and when $B<0$ and $C<0$. The former inequalities imply:

$$
\begin{array}{r}
b_{1} d_{2} \delta_{4}\left(\beta_{11} m_{1}+\beta_{12}\right)+b_{2} d_{1} \delta_{4}\left(\beta_{21} m_{1}+\beta_{22}\right) \\
>d_{1} d_{2} d_{3}\left[\left(d_{5}+\gamma_{1}+\sigma_{1}\right) m_{1}-\sigma_{2}\right] \\
>d_{1} d_{2} d_{3} \frac{\left(\beta_{11} m_{1}+\beta_{12}\right)\left(\beta_{21} m_{1}+\beta_{22}\right)\left(b_{1}+b_{2}\right) \delta_{4}}{d_{1} d_{3}\left(\beta_{21} m_{1}+\beta_{22}\right)+d_{2} d_{3}\left(\beta_{11} m_{1}+\beta_{12}\right)}
\end{array}
$$

Therefore the conditions $B>0$ and $C<0$ are verified if

$$
\begin{array}{r}
b_{1} d_{2} d_{3}\left(\beta_{11} m_{1}+\beta_{12}\right)^{2}+b_{2} d_{1} d_{3}\left(\beta_{21} m_{1}+\beta_{22}\right)^{2} \\
>b_{2}\left(1-d_{1} d_{3}\right)\left(\beta_{11} m_{1}+\beta_{12}\right)\left(\beta_{21} m_{1}+\beta_{22}\right)
\end{array}
$$


Finally, with similar arguments as in the case $B, C>0$, it can be stated the condition for which the last situation occurs, that is $B<0$ and $C<0$ :

$$
\left[\left(d_{5}+\gamma_{1}+\sigma_{1}\right) m_{1}-\sigma_{2}\right]<\min \left[B_{1}, C_{1}\right]
$$

From all this analysis it can be deduced that the proposed measles epidemic spread model allows at most two equilibrium points, $P_{e 1}$ and the solution, if it exists and is admissible, of equation (25).

Finally, to establish the stability of the determined equilibrium point(s) the jacobian matrix is correspondingly evaluated to determine the eigenvalues; in the numerical Section this calculus is provided for the proposed choice of parameters.

\subsection{The Optimal Control Problem}

The natural framework in which epidemic control is faced is the optimal control theory that allows to allocate the limited resources in an efficient way.

The control strategies proposed in this paper are the preventions and the treatments; the former include the most important one, the vaccination, whereas the latter range from the sensibilization to enter in the quarantine condition to the treatment specific for the infectious disease, and for the complication.

The idea is to choose the control (9) in such a way that the number of infected patients, both with and without complications, is minimized. The proposed cost index is:

$$
\begin{aligned}
J= & \frac{1}{2} \int_{t_{0}}^{t_{f}} L(X, U, t) d t \\
= & \frac{1}{2} \int_{t_{0}}^{t_{f}}\left(A_{1} I_{1}^{2}+A_{2} I_{2}^{2}+B_{1} u_{V}^{2}+B_{2} u_{P 1}^{2}+B_{3} u_{P 2}^{2}\right. \\
& \left.+B_{4} u_{T 1}^{2}+B_{5} u_{T 2}^{2}+B_{6} u_{T 3}^{2}+B_{7} u_{T 4}^{2}\right) d t
\end{aligned}
$$

In view of the use of the Pontryagin principle, the Hamiltonian function is defined as:

$$
\begin{aligned}
H & =\frac{1}{2} L(X, U, t)+\lambda^{T}(t) F(X, U, t) \\
& =\frac{1}{2}\left[\sum_{i=1}^{2} A_{i} I_{i}^{2}+\sum_{j=1}^{6} B_{i} U_{i}^{2}\right]+\lambda^{T}(t) F(X, U, t)
\end{aligned}
$$

where $\lambda_{i}, i=1,2, \ldots, 7$ are the costate functions. The necessary conditions of optimality are given by:

$$
\begin{array}{cc}
\dot{\lambda}_{i}=-\frac{\partial H}{\partial X_{i}}, & i=1,2, \ldots, 7 \\
0=\frac{\partial H}{\partial u_{j}}, & j=1, . ., 7
\end{array}
$$

with final conditions:

$$
\lambda_{i}\left(t_{f}\right)=0, \quad i=1,2, \ldots, 7
$$

By solving conditions (38) and taking into account the constraints (13), along with the (37), the optimal control $U_{i}, i=1, \ldots, 7$ are determined:

$$
\begin{aligned}
u_{V} & =\max \left\{\min \left\{\frac{\left(\lambda_{1}-\lambda_{7}\right) \delta_{1} S_{1}}{B_{1}}, U_{1}^{M}\right\}, 0\right\}(40) \\
u_{P 1} & =\max \left\{\min \left\{\frac{-\lambda_{1} \delta_{2} S_{1}}{B_{2}}, U_{2}^{M}\right\}, 0\right\} \\
u_{P 2} & =\max \left\{\min \left\{\frac{-\lambda_{2} \delta_{3} S_{2}}{B_{3}}, U_{3}^{M}\right\}, 0\right\} \\
u_{T 1} & =\max \left\{\min \left\{\frac{\left(\lambda_{5}-\lambda_{4}\right) \delta_{4} E}{B_{4}}, U_{4}^{M}\right\}, 0\right\} \\
u_{T 2} & =\max \left\{\min \left\{\frac{\left(\lambda_{4}-\lambda_{7}\right) \delta_{5} Q}{B_{5}}, U_{5}^{M}\right\}, 0\right\}(43) \\
u_{T 3} & =\max \left\{\min \left\{\frac{\left(\lambda_{5}-\lambda_{7}\right) \delta_{6} I_{1}}{B_{6}}, U_{6}^{M}\right\}, 0\right\} \\
u_{T 4} & =\max \left\{\min \left\{\frac{\left(\lambda_{6}-\lambda_{7}\right) \delta_{7} I_{2}}{B_{7}}, U_{7}^{M}\right\}, 0\right\}(46)
\end{aligned}
$$

Note that the optimal controls require the solution of the costate equations (37) with the final condition (39) in addition to the state equation (11) with the initial conditions (12).

\section{NUMERICAL RESULTS AND DISCUSSION}

In this Section, a numerical analysis is performed to study the proposed measles epidemic model and to evaluate the effects of different control strategies in a fixed time period $[0,30]$ years. As far as the choice of the model parameters, they are strictly dependent on the specific population on which the model is referred to: different healthy, economic and social conditions could vary significantly its numerical values. In all the cases analysed the initial conditions are set equal to $X_{0}=\left(\begin{array}{lllllll}900 & 10 & 5 & 1 & 1 & 0 & 0\end{array}\right)^{T}$, whereas for the model parameters the values

$$
\begin{aligned}
& b_{1}=10, \quad b_{j}=1, j=2, \ldots, 5, \quad b_{6}=0, \\
& d_{1}=d_{7}=0.015, \quad d_{2}=0.02, \\
& d_{3}=d_{4}=0.05, \quad d_{5}=0.08, \quad d_{6}=0.09 \\
& \beta_{i j}=0.01, i=1,2, j=1,2, \\
& \sigma_{1}=0.4, \quad \sigma_{2}=0.2, \\
& \gamma_{1}=\gamma_{3}=0.1, \quad \gamma_{2}=0.07 \\
& \delta_{1}=0.9, \quad \delta_{2}=\delta_{3}=0.2, \quad \delta_{j}=0.4, j=4, . .7
\end{aligned}
$$

are assumed. Note the higher values of death parameters for the subjects in the $I_{1}$ and $I_{2}$ classes corresponding to the risky condition of being infected and, most of all, of being infected with a complication. 
The parameter $\sigma_{1}$ is assumed larger than $\sigma_{2}$ to stress the larger rate of getting the complication rather than recovering from it. By using the analsyis of Subsection 2.2, the obtained equilibrium points for the proposed choice of parameters are:

$$
\left.P_{e 1}=\left(\begin{array}{lllllll}
667 & 50 & 0 & 0 & 0 & 0 & 0
\end{array}\right)^{T}\right)^{T}
$$

always present, and:

$$
P_{e 2}=\left(\begin{array}{lllllll}
2 & 0 & 219 & 0 & 245 & 272 & 2906
\end{array}\right)^{T}
$$

These results are consistent with what stated previously; in fact by evaluating the parameters $A, B$, and $C$ given by (26), (27) and (28) respectively, it is found:

$$
A=10^{-6}, \quad B=-0.0016, \quad C=-0.0016
$$

Therefore a second equilibrium point, besides $P_{e 1}$, exists. The evaluation of the Jacobian matrix in each of these points and the determination of the corresponding eigenvalues allow to state that $P_{e 1}$ is not stable, whereas the $P_{e 2}$ is locally asymptotically stable, having all the eigenvalues real and negative.

The optimal control actions proposed are introduced in the model and compared with three situations: free evolutions (Case 1), only constant vaccination (Case 2), only constant prevention campaign (without vaccination) with constant treatments (Case $3)$. The comparison is proposed both from graphical, Figs. 2-8, and numerical, Table 1 and Table 2, point of view. The indicators introduced aim at evaluating the effectiveness of the actions with respect to the peculiarities of the epidemic disease considered, that is to avoid susceptible subjects $S_{2}$ to get the infections and to avoid infected subjects $I_{1}$ to get the complication, thus entering in the $I_{2}$ class. The optimal control strategy is referred as Case 4 . The indicators proposed in Table 1 are:

- the percentage of dead patients infected, i.e. the total number of dead patients in the $E, Q, I_{1}$ and $I_{2}$ compartments with respect to the total number of dead people in the population;

- the number $S_{2}^{t f}$ of susceptible subjects in $S_{2}$ at the end of the control period;

- the percentage $\bar{S}_{2}$ of susceptible subjects $S_{2}$ in the entire control period;

- the percentage $\bar{I}_{1}$ of infected patients $I_{1}$ in the entire control period;

- the percentage $\bar{I}_{2}$ of infected patients $I_{2}$ in the entire control period;

- the percentage $\bar{R}$ of removed individuals $R$ in the entire control period.
Moreover, also the cost of each strategy is evaluated by considering the total control effort $\bar{U}_{j}$ of each $U_{j}$, see Table 2.

As far as the control actions, they are assumed bounded between 0 and 0.8 . In the cost index the control actions are weighted, aiming at an efficient resources allocation; the solution of the optimal control problem depends on the choice of the parameters in the cost index, as usual; the proposed setting corresponds to the need of allowing a deeper prevention campaign trying to avoid as much as possible the risky condition of the infection. Therefore, the following choices are proposed:

$$
B_{i}=10^{-4}, i=1,2,3 \quad B_{j}=0.01, i=4, . ., 7
$$

Hence, the treatments are considered more expensive than the prevention controls. As far as the parameters of the optimal control problem formulations, they have been chosen as $A_{1}=0.1$ and $A_{2}=10$; this means that the aim is to minimize mainly the number of infected subjects $I_{2}$, being the ones that are risking more with the infection and the complication. Of course other choices are possible; in this case it is stressed the dangerousness of being in the $I_{2}$ class. In Figs. 2-8 the evolutions of the state variables in the four cases considered are proposed. The increased number of $S_{2}$ subjects is evident, as well as the decrease of the infected subjects (patients in $E, Q, I_{1}$ and $I_{2}$ ).

The Case 3, devoted to the treatments, allows a large number of people to enter in the quarantine condition, sensibly more than in Case 4; this is reasonable, since in the latter, being possible all the control actions together, it has been chosen to weight more the treatments, assuming more costly (also from a social point of view) to act after the infection, rather than preventing it. It it worth to be noted the evolution of the removed subjects: in the non controlled case (Case 1 ) the population will enter in the $R$ class, sooner or later; with the optimal control the idea is to avoid people to enter in the epidemic path ( $E, Q$ or $I_{1}$ and maybe $I_{2}$ ) and getting the immunity or remaining in the susceptible condition $S_{2}$, reducing the number of dead patients (see Table 1). Therefore the evolution of the $R$ subjects is rather constant in Case 4.

As far as the optimal control actions it is worth to be noted that the vaccination is required at its upper bound for most of the control period, as well as the control $u_{T 1}$, that induces patients to quarantine, thus avoiding to infect susceptible people. This is reasonable, having chosen to minimize the number of infected patients.

From Table 1 it is evident the advantage of allocating efficently the resources; in fact the number of dead people is one order lower in Case 4 with respect 
to the absence of control (Case 1); the decrease is evident also with respect to constant controls applied, Case 2 (control only by the vaccination) and Case 3 (control only by the prevention, without vaccination, and treatments).

The advantages in Case 4 are evident when considering the number of susceptible people $S_{2}$ at the end of the control period; moreover also the number of recovered people, that is immunized subjects (where the immunization is obtained by vaccination or after having caught the disease), strongly increases in Case 4. Obviously, the efficient use of all the available controls is more expensive, rather than do not do nothing (Case 1) or use only some of the control actions, as evidenced in Table 2.

It is interesting to note what happen if in the cost index the weights of the control are increased of two orders of magnitude, meaning that it becomes more expensive (for all the controls) to minimize the infected patients $I_{1}$ and $I_{2}$. The numerical values are shown in Table 1 and Table 2, indicated as Case 5; it is important to note that from a numerical point of view the results appear rather equivalent, slightly less expensive than in the Case 4, see Table 2. What is interesting is the behaviour of the controls $u_{V}, u_{P 1}$ and $u_{P 2}$, as shown in Figs. (12), (13), (14). In fact, while the evolutions of the treatments control do not vary significantly, in Case 5 the vaccination is at its maximum value for less than five years, with a required increase of the other two prevention controls $u_{P 1}$ and $u_{P 2}$. Therefore, the optimal control formulation allows to allocate optimally the more expensive resources obtaining almost comparable numerical results for the indicators, as noted.

As noted, the results of the optimal control strategy depend on the weights $A_{i}, i=1,2$ and $B_{j}, j=$ $1, \ldots, 7$. The choice proposed in Case 4 and Case 5 aims at reducing mainly the number of patients $I_{2}$, being the ones more at risk. Since in the model it is assumed that a subjects becomes a member of the risky class $I_{2}$ only after entering in the $I_{1}$ class, a strategy could be to avoid strongly the infection, and thus assigning an higher weight to $A_{1}$, than to $A_{2}$. Therefore, in the following Case 6 it assumed $A_{1}=10, A_{2}=0.1$, with the same values of the weight $B_{j}, j=1, \ldots, 7$ as in Case 4. It can be noted the slight increase of the percentage of dead patients with respect to Case 4 but the almost equivalent results for the other indicators of Table 1 . The cost of the vaccination increases, while there is a sensible decrease in the prevention actions $u_{P 1}$ and $u_{P 2}$, see Table 2; this result could be justified, considering that, besides any complication, the best strategy to avoid people to get the measles the most effective strategy is the vaccination.

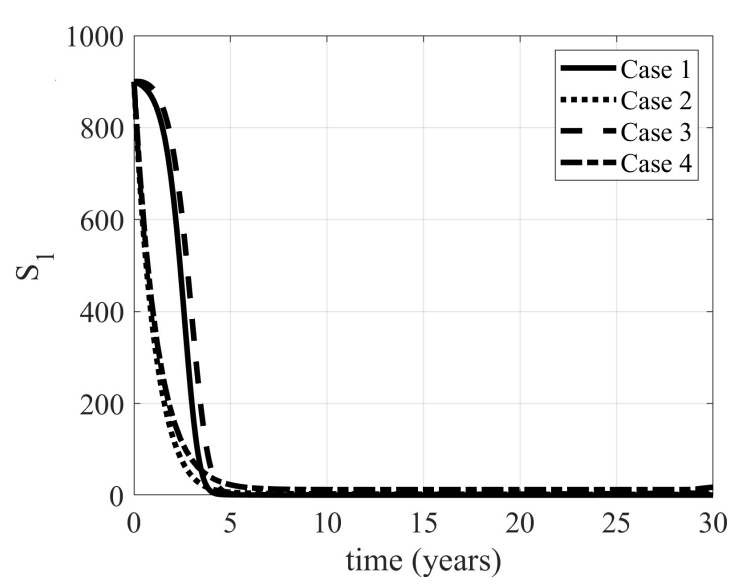

Figure 2: Evolutions of the $S_{1}$ subjects in Cases 1,2,3,4.

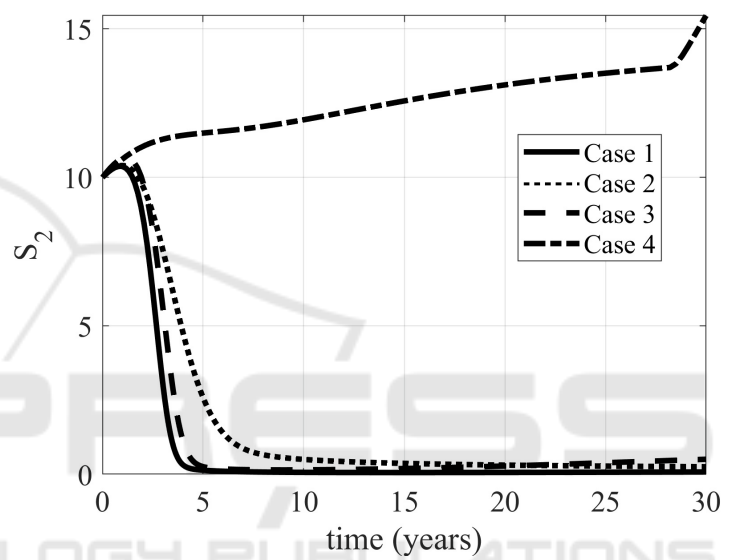

Figure 3: Evolutions of the $S_{2}$ subjects in Cases 1,2,3,4.

Table 1: Evaluation of the cases proposed.

\begin{tabular}{|c||c|c|c|c|c|c|}
\hline & $\begin{array}{c}\text { Dead } \\
(\%)\end{array}$ & $S_{2}^{t f}$ & $\begin{array}{c}\overline{S_{2}} \\
(\%)\end{array}$ & $\begin{array}{c}\overline{I_{1}} \\
(\%)\end{array}$ & $\begin{array}{c}\overline{I_{2}} \\
(\%)\end{array}$ & $\begin{array}{c}\bar{R} \\
(\%)\end{array}$ \\
\hline \hline Case 1 & 87.7 & 0 & 0.03 & 19.6 & 19.9 & 37.9 \\
\hline Case 2 & 60.5 & 0 & 0.28 & 7.8 & 7.6 & 59.6 \\
\hline Case 3 & 78.8 & 1 & 0.12 & 11.6 & 9.18 & 44.6 \\
\hline Case 4 & 7.98 & 15 & 1.38 & 0.25 & 0.3 & 90.7 \\
\hline Case 5 & 8.17 & 21 & 1.51 & 0.26 & 0.3 & 90.4 \\
\hline Case 6 & 8.00 & 15 & 1.38 & 0.25 & 0.3 & 90.8 \\
\hline
\end{tabular}

\section{CONCLUSIONS}

The measles infectious disease represents a risk for subjects that can not be vaccinated, mainly for the possible complications. In this paper this problem is faced by proposing a new model that extends the classical SEIR scheme usually adopted introducing the specific class of immunosuppressed subjects and the one of subjects infected with complication. The aim is to determine the best global control strategy includ- 


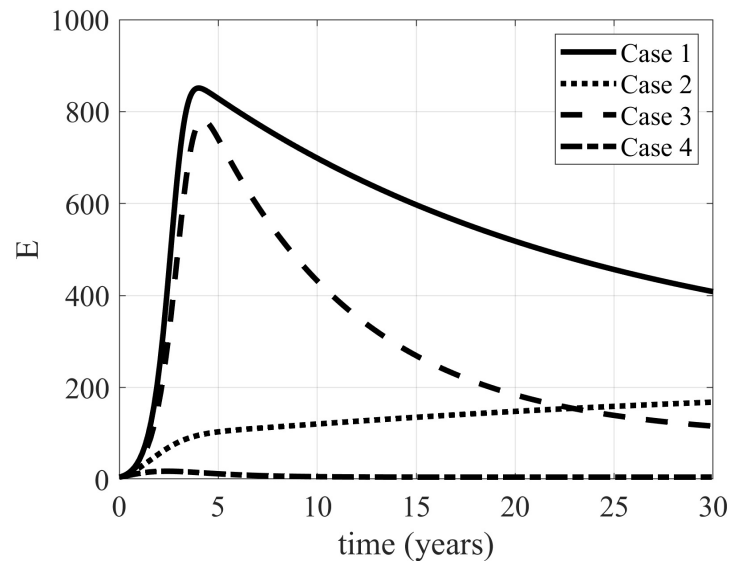

Figure 4: Evolutions of the $E$ subjects in Cases 1,2,3,4.

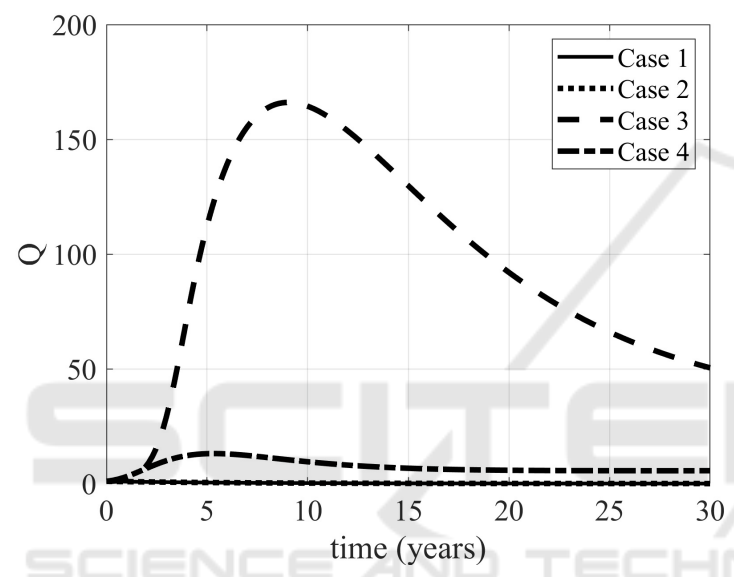

Figure 5: Evolutions of the $Q$ subjects in Cases 1,2,3,4.

Table 2: Evaluation of the control strategies.

\begin{tabular}{|c||c|c|c|}
\hline & $\bar{U}_{1}$ & $\sum_{2}^{3} \bar{U}_{j}$ & $\sum_{4}^{7} \bar{U}_{j}$ \\
\hline \hline Case 1 & & & \\
\hline Case 2 & 24 & & \\
\hline Case 3 & & 6 & 24 \\
\hline Case 4 & 23.58 & 1.1 & 82.42 \\
\hline Case 5 & 21.6 & 9.1 & 81.28 \\
\hline Case 6 & 23.75 & 0.61 & 82.32 \\
\hline
\end{tabular}

ing vaccination, other prevention actions (such as informative campaign) and treatments. In the framework of optimal control theory and under the hypothesis of limited resources, an optimal solution to the problem of decreasing the number of infected patients is proposed. The central role of vaccination is confirmed, along with a synergy among all the introduced actions; the improvements due to the optimal control determined are evident when compared with the absence of control or with strategies that consider treatments and preventions (but not vaccination) or those that introduce only the vaccination. Future work should identify the model parameters referring to spe-



Figure 6: Evolutions of the $I_{1}$ subjects in Cases 1,2,3,4.

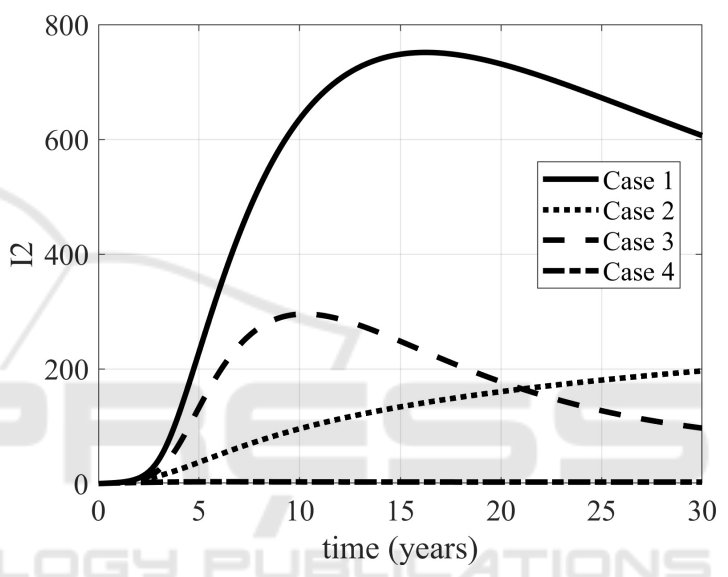

Figure 7: Evolutions of the $I_{2}$ subjects in Cases 1,2,3,4.

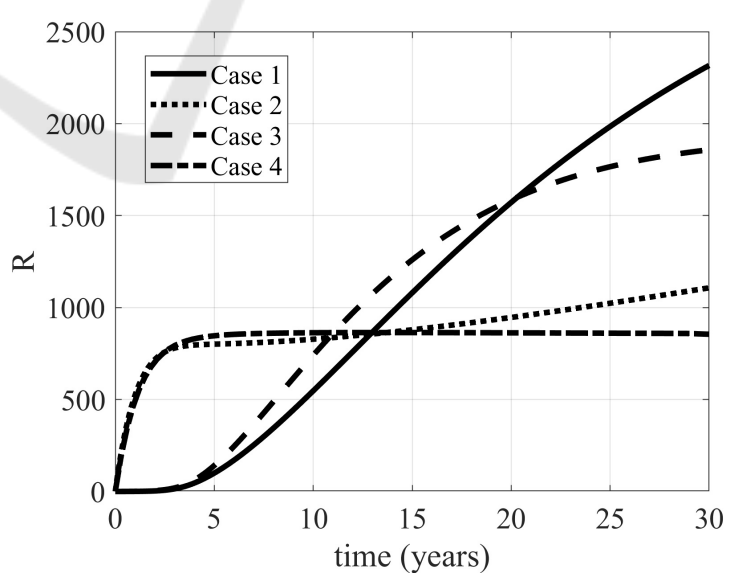

Figure 8: Evolutions of the $R$ subjects in Cases 1,2,3,4.

cific scenarios, taking into account socio-economics and cultural conditions. Consequently, also the parameters referring to the control actions should be reconsidered taking into account the economic cost of prevention and of treatments. In the proposed formulation the final time is fixed at 30 years; nevertheless 


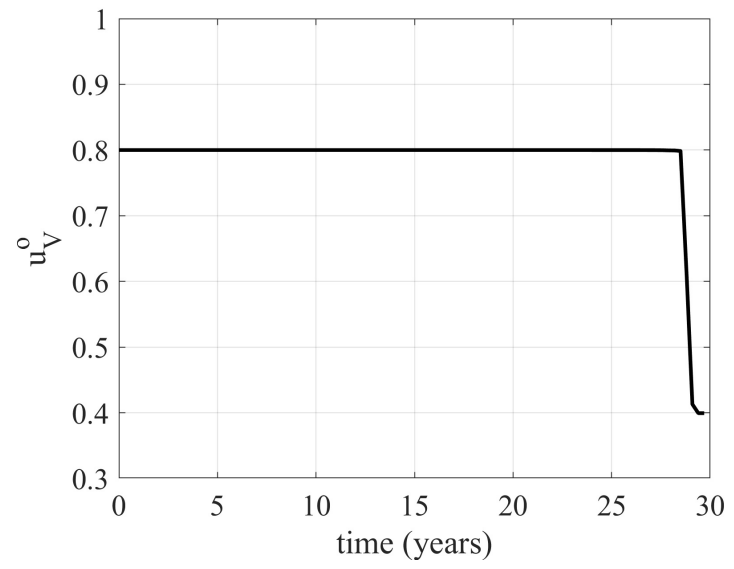

Figure 9: Evolution of optimal vaccination $u_{V}^{o}$.

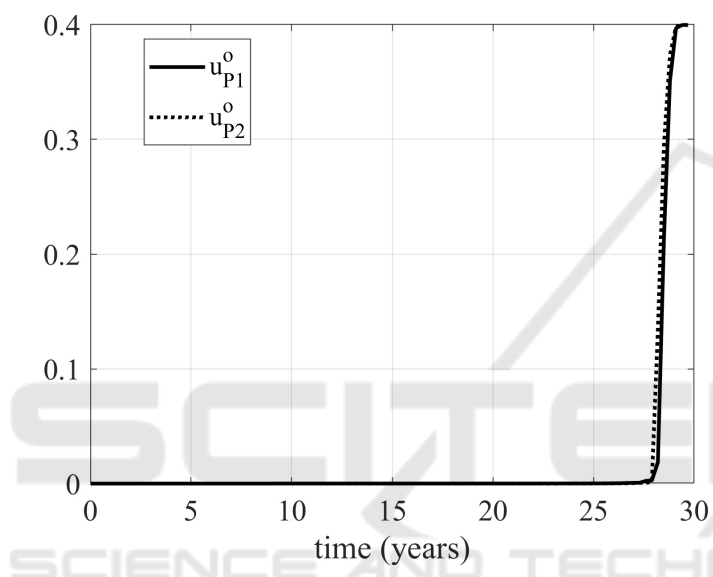

Figure 10: Evolutions of the $u_{P 1}^{o}$ and $u_{P 2}^{o}$ controls.

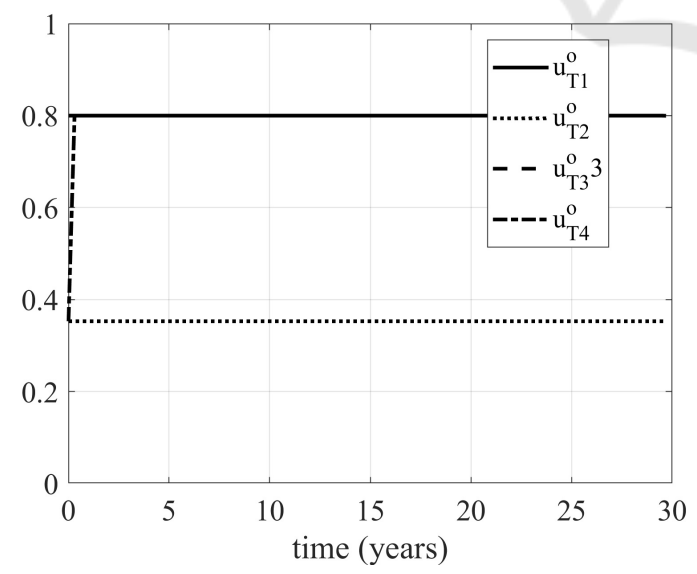

Figure 11: Evolutions of the $u_{T j}^{o}, j=1, . ., 4$ controls.

it could be interesting, referring to particular cases, to determine the best strategy to reduce the number of infected patients in the minimum time.

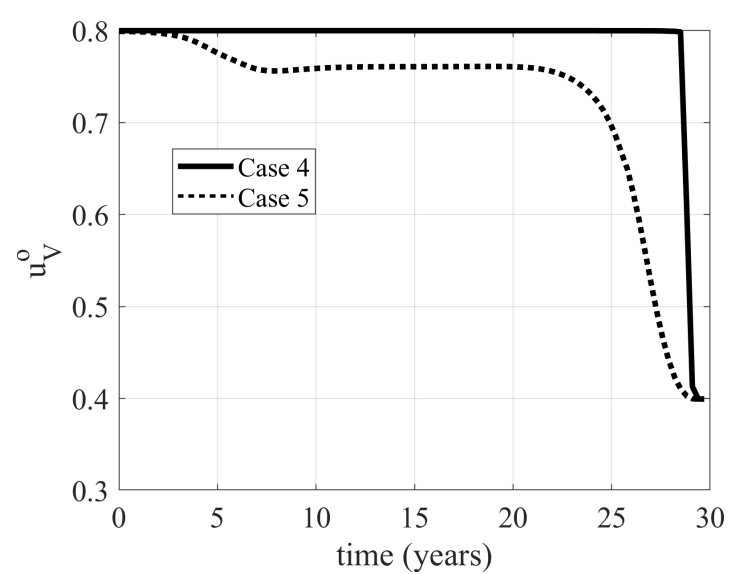

Figure 12: Evolution of the $u_{V}^{o}$ control in Cases 4 and 5.

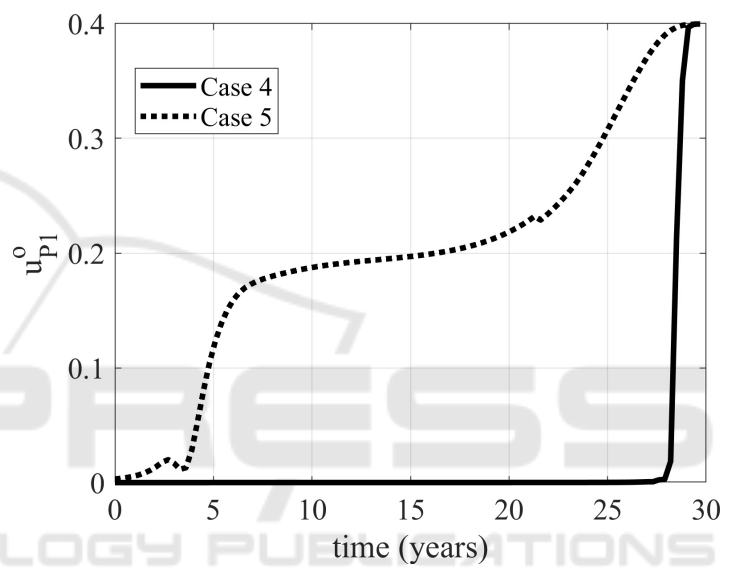

Figure 13: Evolution of the $u_{P 1}^{o}$ control in Cases 4 and 5 .

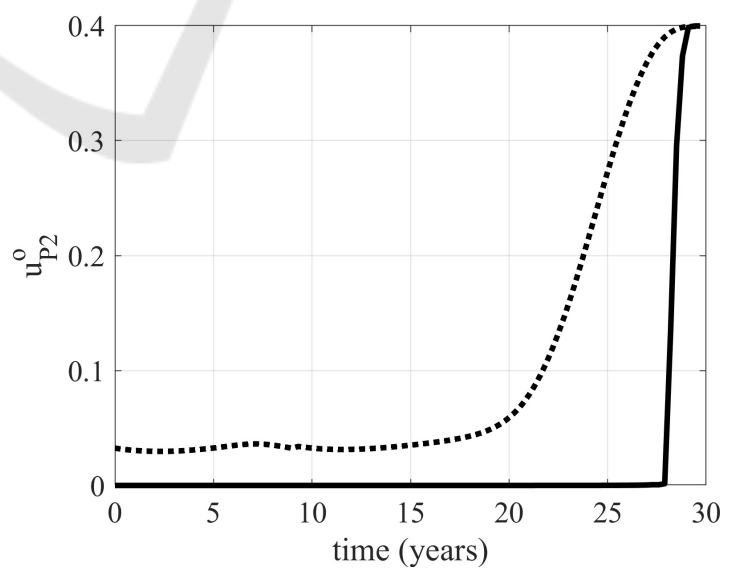

Figure 14: Evolution of the $u_{P 2}^{o}$ control in Cases 4 and 5.

\section{REFERENCES}

A.A.Momoh, Ibrahim, M., i.J.Uwanta, and S.B.Manga (2013). Mathematical model for control of measles 
epidemiology. International Journal of Pure and Applied Mathematics, 87(5):707-718.

Di Giamberardino, P., Compagnucci, L., Giorgi, C. D., and Iacoviello, D. (2018). Modeling the effects of prevention and early diagnosis on hiv/aids infection diffusion. IEEE Transactions on Systems, Man and Cybernetics: Systems.

Di Giamberardino, P. and Iacoviello, D. (2017). Optimal control of SIR epidemic model with state dependent switching cost index. Biomedical Signal Processing and Control, 31.

Di Giamberardino, P. and Iacoviello, D. (2018a). Lq control design for the containment of the hiv/aids diffusion. Control Engineering Practice, 77.

Di Giamberardino, P. and Iacoviello, D. (2018b). Modeling and control of an epidemic disease under possible complication. Proceedings of the 22nd International Conference on System Theory, Control and Computing, pages 67-72.

E.A.J.Fischer, Vlas, S., J.H.Richardus, and Habbema, J. (2008). Musidh, multiple use of simulated demographic histories, a novel method to reduce computation time in microsimulation models of infectious diseases. Computer Methods and Programs in Biomedicine, 91:185-190.

Iacoviello, D. and Stasio, N. (2013). Optimal control for sirc epidemic outbreak. Computer Methods and Programs in Biomedicine.

Kuniya, T. and Nakata, Y. (2012). Permanence and extinction for a nonautonomous seirs epidemic model. $A p$ plied Mathematics and Computation, 218.

Ledzewicz, U. and Schattler, E. (2011). On optimal singular controls for a general SIR-model with vaccination and treatment. Discrete and continuous dynamical systems.

L.Pang, S.Ruan, S.Liu, Z.Zhao, and X.Zhang (2015). Transmission dynamics and optimal control of measles epidemics. Applied mathematics and computations, 256:131-147.

Naresh, R., Tripathi, A., and Sharma, D. (2009). Modeling and analysis of the spread of aids epidemic with immigration of hiv infectives. Mathematical and Computer Modelling, 49.

Nowak, M. and May, R. (2000). Virus Dynamics.

Nowzari, C., Preciado, V. M., and Pappas, G. J. (2016). Analysis and control of epidemics. a survey of spreading processes on complex networks. IEEE Control Systems Magazine.

O.O.Onyejekwe and E.Z.Kebede (2015). Epidemiological modeling of measles infection with optimal control of vaccination and supportive treatment. Applied and computational mathematics, 4(4):264-274.

Pinto, C. and Rocha, D. (2012). A new mathematical model for co-infection of malaria and hiv. 4th IEEE International Conference on Nolinear Science and Complexity.

R.T.Perry and N.A.Halset (2004). The clinical significance of measles: a review. The Journal of Infectious Diseases, 189(1):4-16.
S.O.Adewale, I.A.Olopade, S.O.Ajao, and G.A.Adeniran (2016). Optimal control analysis of the dynamical spread of measles. International Journal of research, 4(5):169-188.

S.Verguet, M.Johri, S.K.Morris, C.L.Gauvreau, P.Jha, and M.Jit (2015). Controlling measles using supplementary immunization activities: a mathematical model to inform optimal policy. Vaccine, 33(10):1291-1296.

TW, N., Turinici, G., and A.Danchin (2003). A double epidemic model for the sars propagation. BMC Infect Dis., 10.

X.Yan and Y.Zou (2008). Optimal and sub-optimal quarantine and isolation control in sars epidemics. Mathematical and computer modelling, 47. 